Washington University School of Medicine Digital Commons@Becker

Open Access Publications

2014

\title{
Total knee arthroplasty after previous knee surgery
}

\author{
Robert H. Brophy \\ Washington University School of Medicine in St. Louis \\ Benjamin L. Gray \\ Washington University School of Medicine in St. Louis \\ Ryan M. Nunley \\ Washington University School of Medicine in St. Louis \\ Robert L. Barrack \\ Washington University School of Medicine in St. Louis \\ John C. Clohisy \\ Washington University School of Medicine in St. Louis
}

Follow this and additional works at: https://digitalcommons.wustl.edu/open_access_pubs Please let us know how this document benefits you.

\section{Recommended Citation}

Brophy, Robert H.; Gray, Benjamin L.; Nunley, Ryan M.; Barrack, Robert L.; and Clohisy, John C., "Total knee arthroplasty after previous knee surgery." The Journal of Bone and Joint Surgery. 96, 10. 801-805. (2014). https://digitalcommons.wustl.edu/open_access_pubs/2946

This Open Access Publication is brought to you for free and open access by Digital Commons@Becker. It has been accepted for inclusion in Open Access Publications by an authorized administrator of Digital Commons@Becker. For more information, please contact vanam@wustl.edu. 


\title{
Total Knee Arthroplasty After Previous Knee Surgery
}

\author{
Expected Interval and the Effect on Patient Age
}

\author{
Robert H. Brophy, MD, Benjamin L. Gray, MD, Ryan M. Nunley, MD, Robert L. Barrack, MD, and John C. Clohisy, MD \\ Investigation performed at the Department of Orthopaedic Surgery, Washington University School of Medicine, St. Louis, Missouri
}

\begin{abstract}
Background: With more than 650,000 knee arthroscopies and 175,000 anterior cruciate ligament reconstructions performed annually in the United States, patients presenting for total knee arthroplasty are increasingly likely to have had previous knee surgery. The purpose of this study was to assess the prevalence of previous knee surgery in patients undergoing total knee arthroplasty and to test the hypothesis that patients with previous knee surgery undergo total knee arthroplasty at a younger age.
\end{abstract}

Methods: All patients undergoing primary total knee arthroplasty over the study period who consented to enroll in a prospective total joint registry were reviewed. Inclusion criteria included a diagnosis of osteoarthritis or posttraumatic arthritis.

Results: Of 1372 patients in the registry, 1286 met inclusion criteria. Twenty-nine percent had a history of knee surgery, and significantly more men (39\%) than women $(24 \%)$ had a history of knee surgery $(p<0.0001)$. Patients with previous knee surgery were significantly younger $(p<0.0001)$ at total knee arthroplasty; the mean age (and standard deviation) was $59 \pm 10$ years for patients with previous knee surgery compared with $66.6 \pm 10.4$ years for patients without previous knee surgery. Patients with a history of ligament reconstruction underwent total knee arthroplasty at a significantly younger age $(p<0.0001)$ than patients with a history of other knee surgery; the mean age (and standard deviation) was $50.2 \pm 9.1$ years for patients with a history of ligament reconstruction and $59.9 \pm 9.6$ years for patients with a history of other knee surgery. Among patients who had not undergone previous knee surgery, women underwent total knee arthroplasty at a significantly younger age $(p<0.001)$ than men; the mean age (and standard deviation) was $65.4 \pm 10.3$ years for women and $69.3 \pm 10$ years for men. However, there was no difference in age between the sexes in those with previous knee surgery; the mean age (and standard deviation) was $58.6 \pm 10.1$ years for women and $59.6 \pm 9.8$ years for men. The average interval (and standard deviation) from previous knee surgery to total knee arthroplasty is $13.1 \pm 12.6$ years, longer in men $(17.7 \pm 13.8$ years) than in women $(9.1 \pm 9.8$ years) $(p<0.0001)$.

Conclusions: Patients with previous knee surgery undergo total knee arthroplasty at a significantly younger age than patients without previous knee surgery, especially men and patients with a history of ligament reconstruction. This may be a factor in the rising demand for total knee arthroplasty. Future investigation to identify those at risk for early total knee arthroplasty after knee surgery and to develop methods to delay or to prevent the need for future total knee arthroplasty in these patients is warranted.

Level of Evidence: Prognostic Level III. See Instructions for Authors for a complete description of levels of evidence.

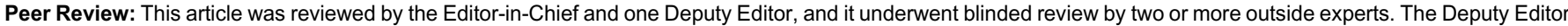

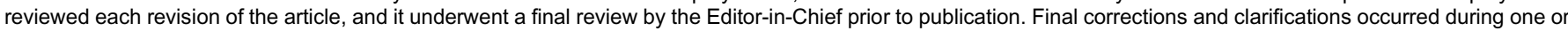
more exchanges between the author(s) and copyeditors.

Disclosure: None of the authors received payments or services, either directly or indirectly (i.e., via his or her institution), from a third party in support of any aspect of this work. One or more of the authors, or his or her institution, has had a financial relationship, in the thirty-six months prior to submission of this work, with an entity in the biomedical arena that could be perceived to influence or have the potential to influence what is written in this work. No author has had any other relationships, or has engaged in any other activities, that could be perceived to influence or have the potential to influence what is written in this work. The complete Disclosures of Potential Conflicts of Interest submitted by authors are always provided with the online version of the article.

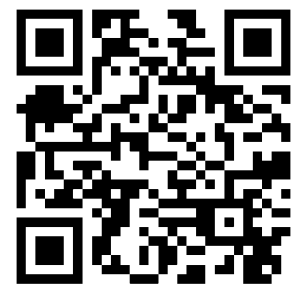

A commentary by Adolph V. Lombardi Jr., MD, is linked to the online version of this article at jbjs.org. 
$\mathrm{T}$ he demand for total knee arthroplasty in the United States is expected to rise substantially in the next twenty years, with a concomitant sharp increase in cost ${ }^{1,2}$. With more than 650,000 knee arthroscopies and approximately 175,000 anterior cruciate ligament (ACL) reconstructions performed annually in the United States ${ }^{3,4}$, patients presenting for total knee arthroplasty are increasingly likely to have had previous knee surgery. Although there is substantial evidence that injuries such as meniscal and ACL tears are associated with a higher risk for developing knee osteoarthritis ${ }^{5-9}$, there are relatively limited data on how this risk translates into the potential need for, and timing of, future total knee arthroplasty.

Patients often inquire as to the likelihood of needing a total knee arthroplasty after knee surgery such as ligament reconstruction or partial meniscectomy. Despite a couple of recent studies looking at the temporal relationship between knee arthroscopy and knee arthroplasty ${ }^{10,11}$, the risk for and timing of total knee arthroplasty after previous knee surgery are not well documented. This is potentially important information to physicians, patients, and health-care systems in terms of understanding the future implications of knee surgery in the adolescent and younger adult populations.

One approach to assess the impact of knee surgery on the need for total knee arthroplasty is to compare the age of patients undergoing total knee arthroplasty with a history of knee surgery to those without a history of knee surgery. This information could provide data to guide patients, physicians, and health-care systems on how knee surgery in younger patients relates to the timing of potential total knee arthroplasty in the future. The purpose of the present study was to assess the prevalence of previous knee surgery in patients undergoing total knee arthroplasty to determine the interval from previous knee surgery to total knee arthroplasty and to test the hypothesis that patients with a history of knee surgery undergo total knee arthroplasty at a younger age compared with those without a history of knee surgery.

\section{Materials and Methods}

A pproval for the study was received from the institutional review board. All $\mathrm{A}_{\text {patients undergoing total knee arthroplasty enrolled in a prospective total }}$ joint registry from 1998 through 2010 were reviewed to select patients with a history of osteoarthritis or posttraumatic arthritis. Patients with a diagnosis of osteonecrosis, failed total knee arthroplasty, or inflammatory arthropathy were excluded. Patient age at the time of the total knee arthroplasty, sex, body mass index (BMI), any previous knee surgery, and the type of knee surgery were recorded. History of knee surgery was collected from patient questionnaires administered as part of the standard of care and included in the registry. This information was cross-checked with patients' medical records as part of the current study to confirm a history of knee surgery and the type of knee surgery. The time from previous knee surgery to total knee arthroplasty, when available, was collected from the medical record.

Summary statistics for age at the time of total knee arthroplasty and BMI were calculated for those with a history of knee surgery and those without a history of knee surgery for the overall cohort and were segregated by patient sex. Subgroups by type of previous knee surgery were also analyzed. Two-sample

\section{Mean age at time of primary total knee replacement in relation to previous knee surgery and patient sex}

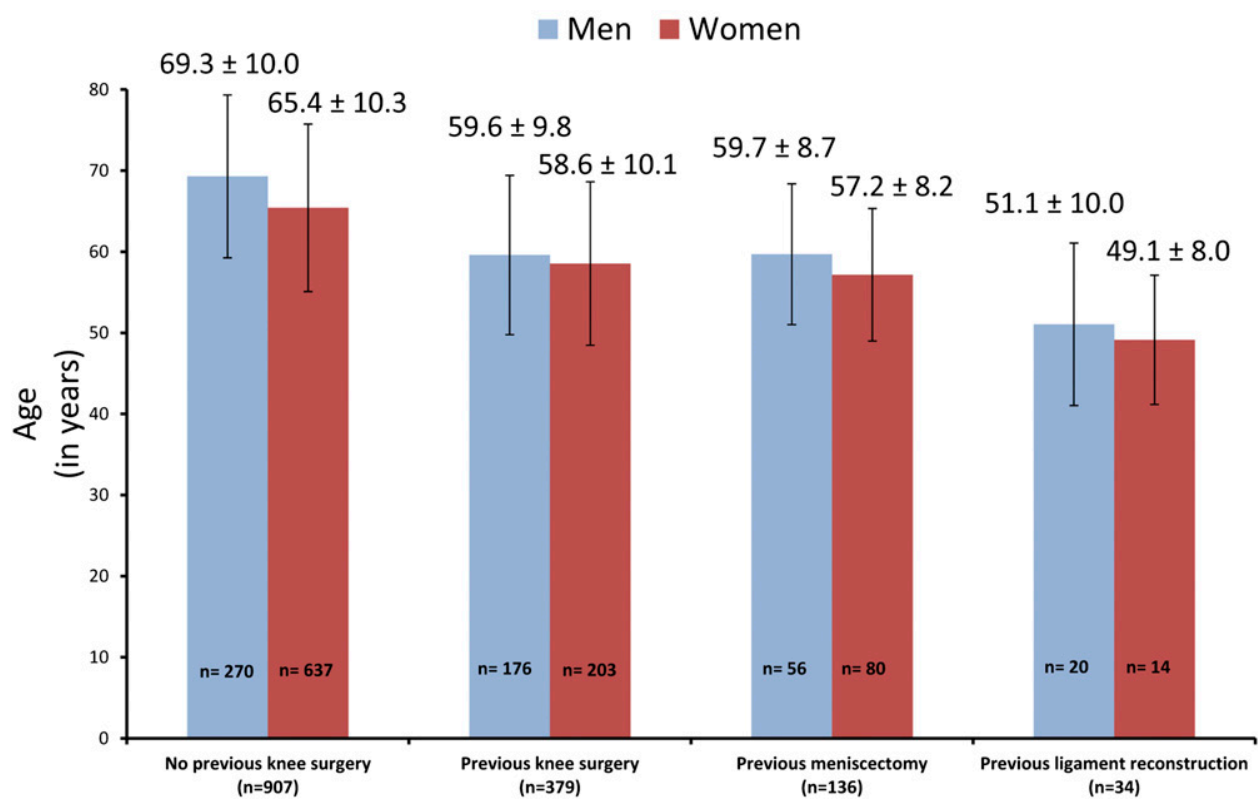

Fig. 1

A bar graph showing the mean age (and standard deviation) at primary total knee arthroplasty in relation to previous knee surgery and patient sex. Men without previous knee surgery were significantly older $(p<0.001)$ than women without previous knee surgery. Patients with previous knee surgery were significantly younger $(p<0.0001)$ than patients without previous knee surgery. Patients with previous ligament reconstruction were significantly younger $(p<0.0001)$ than patients with other previous knee surgery. The error bars indicate the standard deviations. Note that the categories may overlap and do not add up to the total cohort population. 
$\mathrm{t}$ tests were used to compare age at the time of total knee arthroplasty and BMI between patients with previous knee surgery and those without, as well as between different types of previous knee surgery. Summary statistics for the time from the previous surgery to the time of the total knee arthroplasty were recorded for the patients with this information available. Two-sample $t$ tests were used to compare the time from the previous knee surgery to the time of the total knee arthroplasty between different types of previous knee surgery.

\section{Source of Funding}

There was no source of external funding for this study.

\section{Results}

$\mathrm{O}$ f 1372 patients undergoing total knee arthroplasties in the registry, 1286 were undergoing total knee arthroplasties for osteoarthritis or posttraumatic arthritis. Nearly one-third $(29 \%)$ of these patients had a history of knee surgery. The most common type of surgery was arthroscopy $(86 \%)$, followed by meniscectomy (36\%), ligament reconstruction $(9 \%)$, open reduction and internal fixation (9\%), chondroplasty (4.5\%), tibial tubercle transfer (3\%), and osteotomy (3\%) (the percentages do not add up to $100 \%$, as patients may have had more than one type of previous knee surgery).

A greater percentage of men (39\%) had a history of previous knee surgery when compared with women $(24 \%)(\mathrm{p}<0.0001)$. Men and women had a similar distribution of the type of previous knee surgery.

At the time of the primary total knee arthroplasty, patients with a history of knee surgery were significantly younger $(\mathrm{p}<0.0001)$ than patients without a history of knee surgery; the mean age (and standard deviation) was $59 \pm 10$ years for patients with a history of knee surgery and $66.6 \pm 10.4$ years for patients without a history of knee surgery. Among patients who had not undergone previous knee surgery, women underwent total knee arthroplasty at a significantly earlier age $(p<0.001)$ than men; the mean age (and standard deviation) was $65.4 \pm 10.3$ years for women and $69.3 \pm 10$ years for men. However, there was no difference in age between the sexes in those with a history of knee surgery; the mean age (and standard deviation) was $58.6 \pm 10.1$ years for women and $59.6 \pm 9.8$ years for men (Fig. 1). Patients with a history of ligament reconstruction underwent total knee arthroplasty at a significantly younger age $(\mathrm{p}<0.0001)$ than patients with a history of other knee surgery; the mean age (and standard deviation) was $50.2 \pm 9.1$ years for patients with a history of ligament reconstruction and $59.9 \pm 9.6$ years for patients with a history of other knee surgery. Patients who had a history of ligament reconstruction underwent total knee arthroplasty at a similar age, with no significant difference in sex; the mean age (and standard deviation) was $49.9 \pm 9.8$ years for women and $50.4 \pm 8.7$ years for men.

BMI did not appear to have a significant relationship to age at the time of total knee arthroplasty. There was no significant difference in BMI between patients with a history of knee surgery and those without a history of knee surgery (Fig. 2). For patients undergoing total knee arthroplasty with and without a history of knee surgery, men had a lower BMI than women.

Data on the timing of previous knee surgery were available for 309 patients $(81.5 \%)$. The average interval (and standard deviation)

\section{Mean BMI at time of primary total knee replacement in relation to previous knee surgery and patient gender}

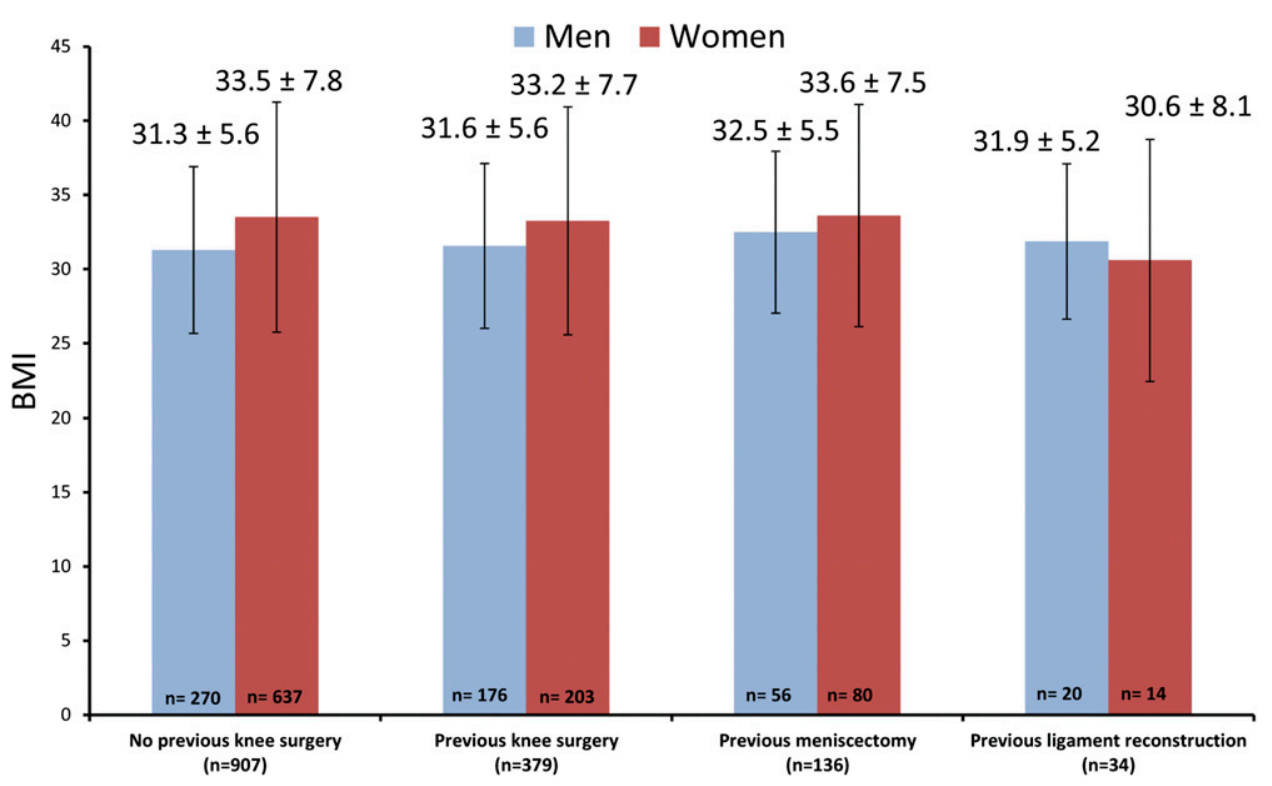

Fig. 2

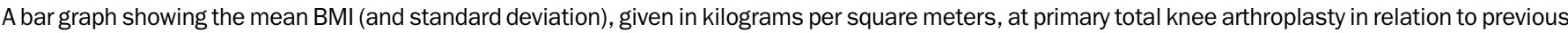

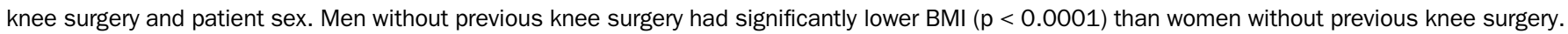
Men with previous knee surgery had significantly lower BMI $(p=0.016)$ than women with previous knee surgery. The error bars indicate the standard deviations. Note that the categories may overlap and do not add up to the total cohort population. 
from previous knee surgery to total knee arthroplasty was $13.1 \pm$ 12.6 years. There was a significant difference $(\mathrm{p}<0.0001)$ between sexes in the interval from the previous knee surgery to the time of the total knee arthroplasty; the mean interval (and standard deviation) was $17.7 \pm 13.8$ years for men and $9.1 \pm 9.8$ years for women. Eighty-nine percent of patients had an interval of more than one year after the previous knee surgery when they underwent total knee arthroplasty. There was no significant difference $(\mathrm{p}=0.097)$ between men $(93 \%)$ and women $(87 \%)$ in the percentage of patients who had an interval of more than one year after knee surgery at the time of total knee arthroplasty.

Patients who had undergone previous meniscectomy underwent total knee arthroplasty at an average interval (and standard deviation) of $12.6 \pm 12.7$ years after meniscectomy. The average interval (and standard deviation) after previous meniscectomy was significantly longer $(\mathrm{p}=0.002)$ for men $(17.6 \pm$ 14.8 years) than for women ( $9.6 \pm 10.3$ years). Fourteen percent of patients underwent total knee arthroplasty within one year after meniscectomy, with no significant difference between men and women. Patients who underwent total knee arthroplasty within one year after partial meniscectomy did not differ significantly in age or BMI from those who underwent total knee arthroplasty at least one year after partial meniscectomy. The average interval (and standard deviation) from ligament reconstruction to total knee arthroplasty was $21.8 \pm 11.2$ years, which was significantly longer than the interval from meniscectomy $(\mathrm{p}=0.0005)$. There was no significant difference between men and women in the interval from ligament reconstruction to total knee arthroplasty.

\section{Discussion}

Datients with previous knee surgery undergo total knee arthroplasty at a significantly younger age than patients without previous knee surgery, especially for patients with a history of ligament reconstruction. Men are more likely than women to have had previous knee surgery prior to total knee arthroplasty, and the effect of previous knee surgery is more profound on men, as those patients undergo total knee arthroplasty at an average age of more than ten years younger than men without previous knee surgery. The interval from knee surgery to total knee arthroplasty is longer for men than for women, particularly for meniscectomy. The interval from meniscectomy to total knee arthroplasty is shorter than the interval from ligament surgery to total knee arthroplasty.

The implications are concerning given the increasing prevalence of knee surgery, especially ligament reconstruction in young and female patients. Although the rising prevalence of obesity and the advancing age of the population as a whole have led to a greater demand for knee arthroplasty, the increasing volume of ligament and meniscal surgery may also be a factor increasing the demand for knee arthroplasty in a younger population.

Men are particularly affected by previous knee surgery. They are more likely than women to have had previous knee surgery, and it has a more profound effect on their age at the time of primary total knee arthroplasty. Among patients without previous knee surgery, men undergo total knee arthroplasty almost four years later than women. In contrast, women and men with previous knee surgery essentially undergo total knee arthroplasty at the same age. Of particular concern is the early age (a mean of fifty years) at which patients with a history of ligament reconstruction undergo total knee arthroplasty.

However, the implications of previous knee surgery may become even more important for women in the near future. There has been a dramatic increase in female sports participation since the passage of Title IX legislation with a concomitant increase in sportsrelated knee injuries, particularly to the $\mathrm{ACL}^{4}$. Therefore, a greater number of female patients are undergoing knee surgery, especially ligament reconstruction, which could portend a rapid future increase in total knee arthroplasties for younger female patients.

From a societal health-care system perspective, all of these findings are concerning for two reasons. Obviously, having total knee arthroplasty at a younger age increases the demand for total knee arthroplasty in the population overall. Perhaps just as important are the potential implications in terms of increasing the demand for revision total knee arthroplasty. The younger patients are at the time of primary total knee arthroplasty, the more likely they will need revision total knee arthroplasty in the future. Patients with previous knee surgery underwent a total knee arthroplasty at an average age of almost seven years younger than patients without previous knee surgery, and this age difference increased to more than sixteen years younger for patients with a history of ligament reconstruction. This age difference nearly guarantees at least one additional revision over each patient's lifetime.

These findings should not be interpreted as a condemnation of knee surgery, particularly in the adolescent and young adult population. Surgery is a marker for knee injury in the younger patient, not the cause of early knee osteoarthritis. The injuries being treated with surgery, such as meniscal and ligament tears, are the underlying clinical problem. Electing not to treat these injuries with surgery is not an attractive option, nor would it necessarily change the long-term development of osteoarthritis and the need for total knee arthroplasty in these patients ${ }^{7,8}$. For example, patients have a greater risk of developing knee osteoarthritis after an ACL tear with or without ACL reconstruction ${ }^{8}$.

Given the high incidence of meniscal tears in older patient populations ${ }^{12}$, magnetic resonance imaging (MRI) is not indicated in patients over the age of fifty years without mechanical complaints, unless specifically ruling out osteonecrosis. It is imperative to make weight-bearing radiographs in this population prior to arthroscopic partial meniscectomy, which will not be efficacious with substantial joint space narrowing, although a recent prospective, multicenter study demonstrated that arthroscopic partial meniscectomy can be an effective treatment for patients over the age of forty years with a meniscal tear and mild to moderate degenerative changes ${ }^{13}$. Knee arthroscopy is not an effective treatment for knee osteoarthritis ${ }^{14,15}$ and patients should be advised of the low expectation for improvement.

A number of studies have looked at the incidence of and time interval to knee arthroplasty after knee arthroscopy. Johanson et al. ${ }^{11}$ reported an $8.5 \%$ to $10.2 \%$ rate of arthroplasty within one year after arthroscopy in the Medicare population, with rates of $29.5 \%$ to $32.1 \%$ by nine years after arthroscopy. Studies from Canada have shown knee arthroplasty rates of $8.5 \%{ }^{10}$ and $9.2 \%{ }^{16}$ within one year after knee arthroscopy. In our 
population, $14.3 \%$ of patients underwent total knee arthroplasty within one year after arthroscopic meniscectomy. As might be expected, older and obese patients have a higher rate of knee arthroplasty after knee arthroscopy ${ }^{17,18}$. These findings are consistent with our data that patients with a history of knee arthroscopy undergo knee arthroplasty at an earlier age than patients without previous knee surgery, but demonstrate that, even in older populations, not all patients who undergo knee arthroscopy will need knee arthroplasty.

Patients undergoing these surgical procedures should be counseled regarding these findings. Although these findings do not predict the rate of future knee replacement, they do show that these patients are at risk for needing total knee arthroplasty at a younger age than the general population. Patients should be advised to optimize their knee health by maintaining a healthy BMI, strengthening their lower-extremity musculature, and minimizing high-impact activities. More studies should look at how interventions such as medications, injections, and potentially protective surgical procedures, such as realignment surgery or meniscal replacement, could delay the need for total knee arthroplasty.

A major limitation of the current study was the lack of data on patients with previous knee surgery who did not have total knee arthroplasty. Only including the patients who have total knee arthroplasty is a strong selection bias, as there may be subgroups that do very well over time. Therefore, this study does not predict the incidence of future total knee arthroplasty in younger patients undergoing outpatient knee surgery such as partial meniscectomy or ligament reconstruction, nor does it identify risk factors for needing future total knee replacement. As a retrospective study of previous knee surgery, it had potential recall bias, particularly with regard to which type of knee surgery was performed. Many of these patients may have undergone first-generation procedures such as open total meniscectomy and extra-articular ligament reconstructions, and these findings may not be predictive for the impact of current approaches to knee surgery such as meniscal repair and more anatomic ligament reconstruction.
Furthermore, we do not have any data on the time from the previous knee surgery or whether patients with multiple previous procedures are more severely affected than patients with a single previous procedure. Timing may be particularly important for meniscectomy, as the effect of this surgery at a younger age is likely different from the effect of meniscectomy closer to the time of total knee arthroplasty. Finally, we do not have any data on whether a history of knee surgery impacts the clinical outcome and, particularly, the need for revision surgery after total knee arthroplasty.

Despite these limitations, the present study clearly demonstrates that patients who have knee surgery will undergo total knee arthroplasty at a younger age than patients without a history of knee surgery, particularly in men, while women have a shorter interval from knee surgery to total knee arthroplasty. This information may be helpful in counseling patients undergoing knee surgery. The health-care system as a whole needs to be aware of the implications of these findings for increased demand for total knee arthroplasty in younger patients. Future research should investigate whether previous knee surgery affects the outcome of total knee arthroplasty; these factors could predict which patients are likely to need total knee arthroplasty following knee surgery, especially ligament reconstruction, and how to delay the need for total knee arthroplasty in patients who undergo knee surgery in adolescence and early adulthood.

Robert H. Brophy, MD

Benjamin L. Gray, MD

Ryan M. Nunley, MD

Robert L. Barrack, MD

John C. Clohisy, MD

Department of Orthopaedic Surgery,

Washington University School of Medicine,

14532 South Outer Forty Drive,

Chesterfield, MO 63017

\section{References}

1. Kurtz S, Ong K, Lau E, Mowat F, Halpern M. Projections of primary and revision hip and knee arthroplasty in the United States from 2005 to 2030. J Bone Joint Surg Am. 2007 Apr;89(4):780-5.

2. Kurtz SM, Ong KL, Schmier J, Mowat F, Saleh K, Dybvik E, Kärrholm J, Garellick G, Havelin LI, Furnes O, Malchau H, Lau E. Future clinical and economic impact of revision total hip and knee arthroplasty. J Bone Joint Surg Am. 2007 Oct;89(Suppl 3):144-51. 3. Owings MF, Kozak L. Ambulatory and inpatient procedures in the United States, 1996. Vital Health Stat 13. 1998 Nov;(139):1-119.

4. Spindler KP, Wright RW. Clinical practice. Anterior cruciate ligament tear. N Engl J Med. 2008 Nov 13;359(20):2135-42.

5. Englund M. The role of the meniscus in osteoarthritis genesis. Rheum Dis Clin North Am. 2008 Aug;34(3):573-9.

6. Englund M, Guermazi A, Lohmander SL. The role of the meniscus in knee osteoarthritis: a cause or consequence? Radiol Clin North Am. 2009 Jul;47(4):703-12.

7. Lohmander LS, Englund PM, Dahl LL, Roos EM. The long-term consequence of anterior cruciate ligament and meniscus injuries: osteoarthritis. Am J Sports Med. 2007 Oct;35(10):1756-69. Epub 2007 Aug 29.

8. Louboutin H, Debarge R, Richou J, Selmi TA, Donell ST, Neyret P, Dubrana F. Osteoarthritis in patients with anterior cruciate ligament rupture: a review of risk factors. Knee. 2009 Aug;16(4):239-44. Epub 2008 Dec 20.

9. McDermott ID, Amis AA. The consequences of meniscectomy. J Bone Joint Surg Br. 2006 Dec;88(12):1549-56.

10. Hawker G, Guan J, Judge A, Dieppe P. Knee arthroscopy in England and Ontario: patterns of use, changes over time, and relationship to total knee replacement.

J Bone Joint Surg Am. 2008 Nov;90(11):2337-45.
11. Johanson NA, Kleinbart FA, Cerynik DL, Brey JM, Ong KL, Kurtz SM. Temporal relationship between knee arthroscopy and arthroplasty: a quality measure for joint care? J Arthroplasty. 2011 Feb;26(2):187-91. Epub 2010 Jun 11.

12. Englund M, Guermazi A, Gale D, Hunter DJ, Aliabadi P, Clancy M, Felson DT. Incidental meniscal findings on knee MRI in middle-aged and elderly persons. N Engl J Med. 2008 Sep 11;359(11):1108-15.

13. Katz JN, Brophy RH, Chaisson CE, de Chaves L, Cole BJ, Dahm DL, Donnell-Fink LA, Guermazi A, Haas AK, Jones MH, Levy BA, Mandl LA, Martin SD, Marx RG, Miniaci A, Matava MJ, Palmisano J, Reinke EK, Richardson BE, Rome BN, Safran-Norton CE, Skoniecki DJ, Solomon DH, Smith MV, Spindler KP, Stuart MJ, Wright J, Wright RW, Losina E. Surgery versus physical therapy for a meniscal tear and osteoarthritis. N Engl J Med. 2013 May 2;368(18):1675-84. Epub 2013 Mar 18.

14. Kirkley $A$, Birmingham TB, Litchfield RB, Giffin JR, Willits KR, Wong CJ, Feagan BG, Donner A, Griffin SH, D'Ascanio LM, Pope JE, Fowler PJ. A randomized trial of arthroscopic surgery for osteoarthritis of the knee. N Engl J Med. 2008 Sep 11;359(11):1097-107. 15. Moseley JB, O'Malley K, Petersen NJ, Menke TJ, Brody BA, Kuykendall DH, Hollingsworth JC, Ashton CM, Wray NP. A controlled trial of arthroscopic surgery for osteoarthritis of the knee. N Engl J Med. 2002 Jul 11;347(2):81-8.

16. Wai EK, Kreder HJ, Williams Jl. Arthroscopic débridement of the knee for osteoarthritis in patients fifty years of age or older: utilization and outcomes in the Province of Ontario. J Bone Joint Surg Am. 2002 Jan;84(1):17-22.

17. Rand JA. Arthroscopic management of degenerative meniscus tears in patients with degenerative arthritis. Arthroscopy. 1985;1(4):253-8.

18. Spahn G, Mückley T, Kahl E, Hofmann GO. Factors affecting the outcome of arthroscopy in medialcompartment osteoarthritis of the knee. Arthroscopy. 2006 Nov;22(11):1233-40. 